\title{
Conformación fascicular de los nervios tibial y peroneo común en el perro*
}

\author{
Llano, E.G.; Flores Quintana, C.I.; Cabrera, W.R.; Báez, A.D.
}

Cátedra de Anatomía Comparada II Parte, Facultad de Ciencias Veterinarias, UNNE, Sargento Cabral 2139, Corrientes (3400), Argentina, Tel7Fax 03783-425753. E-mail: anato2@vet.unne.edu.ar.

\begin{abstract}
Resumen
Llano, E.G.; Flores Quintana, C.I.; Cabrera, W.R.; Báez, A.D.: Conformación fascicular de los nervios tibial y peroneo común en el perro. Rev. vet. 19: 2, 135-138, 2008. El objetivo del trabajo fue identificar el número y situación espacial de los fascículos que conforman los nervios tibial y peroneo común en el perro, permitiendo de esta manera su clasificación acorde a modelos de agrupación fascicular. Se trabajó con 20 miembros posteriores de caninos. Los nervios tibial y peroneo común fueron disecados. A partir de cortes transversales de dichos nervios se realizaron preparados histológicos coloreados con hematoxilina y eosina, determinándose por microscopía óptica el número y la distribución de los fascículos, agrupándose las fibras en tres categorías acorde a su diámetro (hasta 4, de 4 a 8 y de 8 a 12 $\mu \mathrm{m})$. Para el nervio tibial fueron identificados trece fascículos, el de mayor diámetro ubicado caudal y medialmente del nervio, resultando que el $40 \%$ de sus fibras correspondió a la primera categoría, $52 \%$ para la segunda y $8 \%$ para la tercera. Para el nervio peroneo común se identificaron tres fascículos, el de mayor diámetro ubicado caudalmente, no diferenciando su posición lateral o medial por tratarse de un nervio aplanado, con una distribución lineal de los fascículos; del análisis de las fibras surgió que el 50\% perteneció a la primera categoría, $35 \%$ a la segunda y $15 \%$ a la tercera.
\end{abstract}

Palabras clave: perro, anatomía, nervio tibial, nervio peroneo común, fascículos.

\begin{abstract}
Llano, E.G.; Flores Quintana, C.I.; Cabrera, W.R.; Báez, A.D.: Fascicular conformation of tibial and common peroneal nerves in the dog. Rev. vet. 19: 2, 135-138, 2008. The objective of this work was to identify the number and disposition of the fascicles that form the tibial and common peroneal nerves in the dog, thus allowing their grouping in fascicles. Posterior limbs of twenty dogs were used. Tibial and common peroneal nerves were dissected, and samples were embedded in paraffin, sectioned, processed by standard histology technique, and stained with hematoxylin and eosin. Number and fascicular distribution of nerves were determined by light microscopy. Fibers were grouped in categories: up to 4 , from 4 to 8 , and from 8 to $12 \mu \mathrm{m}$. For the tibial nerve thirteen fascicles were identify, one with the widest diameter located caudal and medial aspect of the nerve, resulting from the analysis that $40 \%$ of the fibers corresponded to the first category, $52 \%$ for the second one, and $8 \%$ for the third one. For the common peroneal nerve three fascicles were identify, one with widest diameter located caudally, with no differentiation of its lateral and medial aspects because of it flattened shape, with a lineal distribution of the fascicles and for the analysis of the fibers, $50 \%$ were of the first category, $35 \%$ of the second one, and $15 \%$ of the third one.
\end{abstract}

Key words: dog, anatomy, tibial nerve, common peroneal nerve, fascicles.

\section{INTRODUCCIÓN}

Los nervios tibial y peroneo, debido a su tamaño, son las ramas terminales más importantes que se desprenden del nervio isquiático $2,5,8,12,19,20$. El nervio tibial es la rama terminal mayor del nervio isquiático. Cursa distalmente entre los músculos bíceps femoral y semi-

Proyecto SGCYT-UNNE (PI 003/06, Resol. 520/07).

Recibido: 3 octubre 2008 / Aceptado: 12 noviembre 2008 membranoso y continúa a través de la región poplítea entre las dos cabezas del músculo gastronemio. En una forma variable inerva las cabezas medial y lateral del músculo gastronemio y los músculos flexor digital superficial, profundo, poplíteo y tibial caudal. Opuesto a la articulación tarsocrural, el nervio tibial se divide en nervios plantares mediales y laterales.

De las dos ramas terminales del nervio isquiático, el nervio peroneo es la mas pequeña. Cruza distocranealmente la cabeza lateral del gastronemio bajo el bí- 
ceps femoral. Después de recorrer el intersticio entre los músculos flexor digital I largo (porción lateral del flexor digital profundo) y el peroneo largo, se divide en ramas superficial y profunda $6,7,11,14$.

El conocimiento profundo y detallado de los nervios periféricos, desde la anatomía macroscópica hasta su caracterización histológica, así como las ramas que forman en su trayecto y sus relaciones con las estructuras vecinas son claves, entre otras cosas, en las técnicas de bloqueo regional anestésico y en la reparación quirúrgica de los mismos ${ }^{3}$.

En una sección transversal de cualquier nervio periférico se observan los axones o fibras nerviosas y su vaina de mielina circundada por el endoneuro. El perineuro delimita y envuelve cada fascículo. Los grupos de fascículos están encerrados por el epineuro, el cual incluye los vasos intraneurales 10, 16, 22. Los fascículos y el epineuro son estructuras importantes al momento de realizar la sutura y aproximación de los segmentos dañados; estos conocimientos complementados con el uso de técnicas microquirúrgicas, ayudan a mejorar la especificidad de la reinervación y la recuperación funcional de los nervios $4,9,15,18$.

Se considera que los troncos nerviosos periféricos presentan distintos tipos de modelos fasciculares en

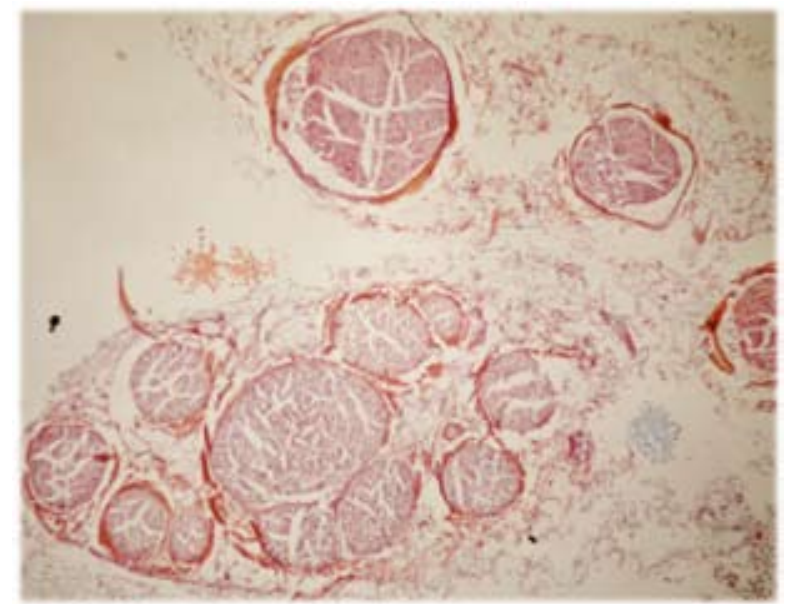

Figura 1. Nervio tibial (10x). Se identifican hasta 13 fascículos.

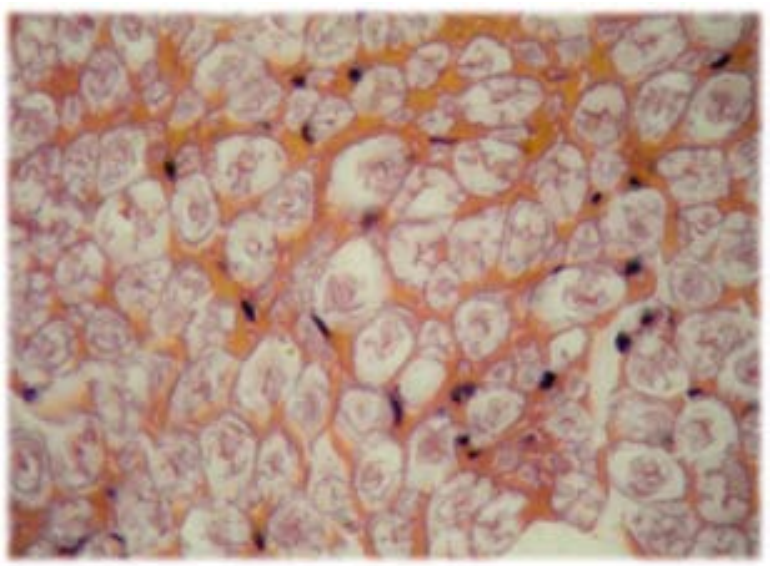

Figura 2. Fibras nerviosas en un fascículo del nervio tibial (100x). distintos nervios y a distintos niveles dentro del mismo nervio, existiendo en anatomía humana una clasificación en modelos de agrupación fascicular. Monofasciculares: compuestos por una única agrupación fascicular; corresponden a nervios unifuncionales como los colaterales digitales o la rama sensitiva cutánea del nervio radial. Oligofasciculares: nervios que agrupan a escasos fascículos; un ejemplo es el nervio cubital durante el recorrido por la muñeca. Polifasciculares con grupos definidos: es el caso de los nervios que presentan numerosos fascículos con agrupación fascicular definida, como el nervio mediano en la muñeca y el nervio radial a nivel del codo. Polifasciculares sin grupos definidos: cuando aparecen abundantes fascículos sin agrupación delimitada; el nervio ciático común cumple estas características ${ }^{1}$.

Los fascículos son relativamente escasos en los nervios periféricos de los animales ${ }^{21}$. Cada nervio periférico está formado por fibras nerviosas que según su función pueden ser motoras o eferentes y sensoriales o aferentes. Por su tamaño las fibras nerviosas varían entre 0,2 a $21 \mu \mathrm{m}$ de diámetro, cuanto más grande es éste, mayor es la velocidad de conducción, el intervalo de velocidad de conducción se encuentra entre 0,5 y $120 \mathrm{~m} / \mathrm{seg}$. Existe una clasificación de uso general de las fibras nerviosas que incluye fibras sensitivas, motoras y asimismo fibras autónomas, en la misma se considera el criterio histológico morfométrico basado en el

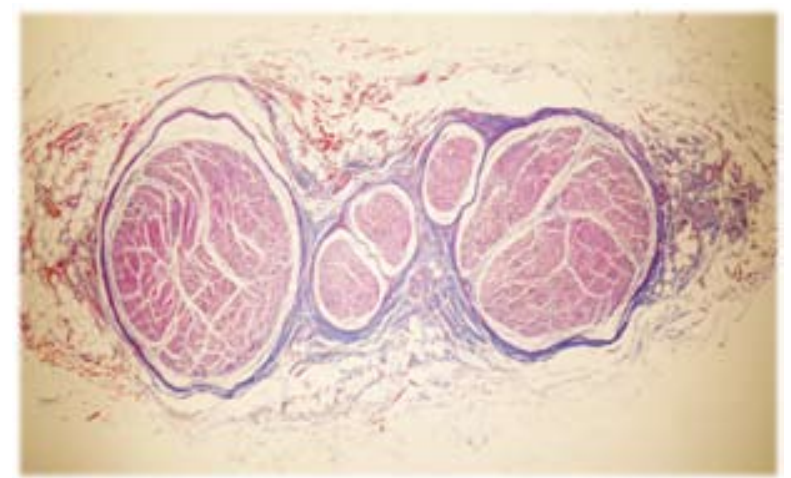

Figura 3. Nervio peroneo común (10x). Se identifican hasta 3 fascículos.

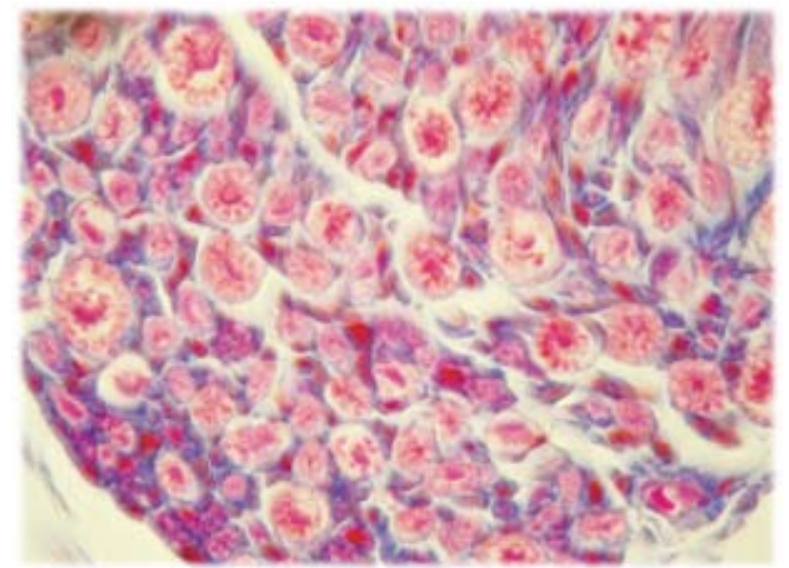

Figura 4. Fibras nerviosas en un fascículo del nervio peroneo común (100x). 
diámetro de las fibras, que a su vez se superpone con el sistema clasificatorio electrofisiológico basado en la velocidad de conducción.

Según el criterio histológico morfométrico, las fibras nerviosas pueden ser: tipo A mielínicas típicas de los nervios espinales, de 1 a $21 \mu \mathrm{m}$, las cuales se subdividen en alfa, beta, gamma y delta. Las fibras tipo A alfa y beta poseen un diámetro de 6 a $21 \mu \mathrm{m}$, con función motora del músculo esquelético, pero también comparten funciones sensitivas en el huso muscular, órgano tendinoso de Golgi, receptores del pelo, vibración (corpúsculo de Pacini) y tacto de alta discriminación (Meissner, terminaciones dilatadas). Las fibras tipo A gamma y delta alcanzan diámetros entre 1 y 6 $\mu \mathrm{m}$ y ejercen la función motora del huso muscular y la función sensitiva de presión, tacto profundo, dolor al pinchazo y sensaciones térmicas. Las fibras $\mathrm{B}$, mielinizadas, miden $1-3 \mu \mathrm{m}$ y constituyen las principales ramas neurovegetativas preganglionares. Las fibras tipo $\mathrm{C}$ son amielínicas, muy pequeñas, no superan los 0,2 a $1 \mu \mathrm{m}$, conducen impulsos a bajas velocidades y constituyen más del $50 \%$ de las fibras sensitivas en los nervios periféricos y todas las fibras posganglionares autonómicas ${ }^{13,17}$.

El objetivo del trabajo fue identificar el número y situación espacial de los fascículos que conforman los nervios tibial y peroneo común en el perro, permitiendo de esta manera su clasificación acorde a modelos de agrupación fascicular.

\section{MATERIAL Y MÉTODOS}

Se utilizaron diez cadáveres caninos, aplicándose la siguiente metodología: 1) laparotomía ventral mediana, evisceración y sección del tren posterior en la región lumbar; 2) fijación del material por inmersión en formol al 10\%; 3) disección clásica de los nervios tibial y peroneo común; 4) sección de los troncos nerviosos en una longitud de $1,5 \mathrm{~cm}$. colocando en ellos un hilo de color que tomó el epineuro longitudinalmente en una extensión de $0,5 \mathrm{~cm}$., el hilo de color rojo indicó la posición lateral, el hilo de color azul la posición craneal; 5) fijación con formol bufferado al $10 \%$ durante 24 hs; 6) inclusión en parafina; 7) realización de cortes con micrótomo tipo Minot a $5 \mu \mathrm{m}$; 8) tinción con hematoxilina y eosina; 9) observación en microscopio óptico identificando el número y distribución fascicular de los nervios en cuestión; 10) determinación del diámetro de 200 fibras en los principales fascículos y agrupación de las mismas en tres categorías, a partir de fotografías obtenidas diferenciadas en zonas periféricas y central de los fascículos, empleando el analizador de imagen Software Image Pro-Plus, versión 4.5, Media Cybernetics Inc.

\section{RESULTADOS Y DISCUSIÓN}

En el nervio tibial se identificaron hasta 13 fascículos de diámetro uniforme, rodeados todos por el epi- neuro; uno de los fascículos era de mayor diámetro y estaba ubicado caudal y medialmente. En el análisis de las fibras se diferenciaron tres categorías: categoría 1 (hasta $4 \mu \mathrm{m}$ de diámetro, donde se encontró el $40 \%$ de las fibras), categoría 2 (de 4 a $8 \mu \mathrm{m}$, donde se encontró el $52 \%$ de las fibras) y categoría 3 (de 8 a $12 \mu \mathrm{m}$, donde se encontró el $8 \%$ de las fibras) (Figuras 1 y 2 ).

En el nervio peroneo común se identificaron 3 fascículos, el de mayor diámetro estaba ubicado caudalmente y no se diferenciaban posiciones lateral o medial por tratarse de un nervio aplanado, con una distribución lineal de los fascículos. En el análisis de las fibras se diferenciaron tres categorías: categoría 1 (hasta 4 $\mu \mathrm{m}$ de diámetro, donde se encontró el $50 \%$ de las fibras), categoría 2 (de 4 a $8 \mu \mathrm{m}$, donde se encontró el $35 \%$ de las fibras) y categoría 3 (de 8 a $12 \mu \mathrm{m}$, donde se encontró el $15 \%$ de las fibras) (Figuras 3 y 4 ).

En la medición de las fibras, ninguna superó el diámetro de $12 \mu \mathrm{m}$, en la bibliografía se citan diámetros de hasta $21 \mu \mathrm{m}$, correspondiendo a las fibras de diámetro mayor a $9 \mu \mathrm{m}$ la función motora del músculo esquelético.

Las descripciones efectuadas sobre la conformación y agrupación fascicular de los nervios tibial y peroneo común en el perro aportan al mejor conocimiento de la anatomía veterinaria y brindan un aporte útil a los profesionales dedicados a la cirugía traumatológica, especialmente en las reparaciones nerviosas.

\section{REFERENCIAS}

1. Ayala Gutiérrez H, Colas C, Palacios JI, Morrondo JC. 2001. Cirugía secundaria de las lesiones de los nervios periféricos. www.secpre.org/documentos.

2. Berg R. 1978. Anatomía topográfica y aplicada de los animales domésticos, Ed. A.C., Madrid, p. 354-378.

3. Bollini C. 2004. Revisión anatómica del plexo braquial. Rev Arg Anest 62: 386-398.

4. Chrisman CH. 1996. Neuropatías periféricas. En: Fisiopatología y clínica quirúrgica en animales pequeños (Bojrab MJ, Ed.), Intermédica, Bogotá, p. 1192-1206.

5. Done SH, Godoy PC, Evans SA, Stickland NC. 1997. Atlas en color de anatomía veterinaria del perro y gato, Harcour Brace, Madrid, p. 7.2-7.37.

6. Dyce KM, Sack WO, Wensing CJ. 1999. Anatomía veterinaria, Panamericana, México, p. 30-32, 338-350.

7. Evans HE, Delahunta A. 2000. Disección del perro, Mc.Graw-Hill, México, p. 160-181, 241-265.

8. Frandson RD. 1976. Anatomía y fisiología de los animales domésticos, Ed. Interamericana, México, p. 57-64.

9. Garibaldi L. 2003. Monoparesias y monoplejias. En: El libro de neurología para la práctica clínica (Pellegrino F, Suraniti A, Garibaldi L, Ed.), Intermédica, Buenos Aires, p. 161-167.

10. Geneser F. 1990. Histología, Panamericana, Madrid, p. 296-298.

11. Getty R. 1982. Anatomía de los animales domésticos, Salvat, Barcelona, p. 209-221, 1862-1887. 
12. Gil J, Gimeno M, Laborda J, Nuviala J. 1997. Anatomía del perro, protocolos de disección, Masson, Madrid, $\mathrm{p}$. 151-191.

13. Guyton AC. 1997. Anatomía y fisiología del sistema nervioso, Panamericana, Madrid, p. 123-125.

14. Konig HE, Liebich HG. 2005. Anatomía de los animales domésticos, Panamericana, Buenos Aires, p. 261-270.

15. Luhers Graca D, Riet-Correa G, Pioli Torres M. 2003. Citología del sistema nervioso periférico y su relevancia en las enfermedades neurológicas. En: El libro de neurología para la práctica clínica (Pellegrino F, Suraniti A, Garibaldi L, Ed.), Intermédica, Buenos Aires, p. 622-626.

16. Marin Gutzke M, Castello Fortet JR, Santos Heredero J. 2001. Lesiones agudas de los nervios periféricos. $w w w$. secpre.org/documentos.

17. Pellegrino F, Sánchez G. 1995. Anatomía funcional del sistema nervioso de los animales domésticos, Ed. AgroVet, Buenos Aires, p.13-37.
18. Pérez Fernández R, Labrador Molina JM, Lara García F, Marcos Vivas A, Hijano Mir JC, Martínez González R, Cabrera Morales E. 2001. Injertos (cutáneos, dermograsos, tendinosos, nerviosos, óseos, cartilaginosos y vasculares). www.secpre.org/documentos.

19. Schaeller O. 1992. Nomenclatura anatómica veterinaria ilustrada, Acribia, Zaragoza, p. 322-325, 494-496.

20. Schwarze E. 1970. Compendio de anatomía veterinaria, Acribia, Zaragoza, p. 78-90.

21. Shores A. 2001. Sistema nervioso y órganos de la sensación. En: Técnicas actuales en cirugía de pequeños animales (Bojrab MJ, Ed.), Intermédica, Montevideo, p. 67-76.

22. Ueyama T. 1978. The topography of root fibres within the sciatic nerve trunk of the dog. J Anat 127: 2, 277-290. 